\title{
Adaptive Visualization using the Annealing M-Estimator
}

\author{
Ruxandra Lasowski ${ }^{1}, 2$, Selim Benhimane ${ }^{1}$, Jakob Vogel ${ }^{1}$, Tobias F. Jakobs ${ }^{3}$, \\ Christoph J. Zech ${ }^{3}$, Christoph Trumm ${ }^{3}$, Martin Brokate ${ }^{4}$, Nassir Navab ${ }^{1}$ \\ ${ }^{1}$ Chair for Computer Aided Medical Procedures (CAMP), TU Munich, Germany \\ ${ }^{2}$ Siemens Medical Solutions, Computed Tomography, Forchheim, \\ ${ }^{3}$ Institute for Clinical Radiology, Grosshadern Hospital, University of Munich, \\ ${ }^{4}$ Technical University Munich, Center of Mathematics, TU Munich, Germany \\ micu@cs.tum.edu
}

\begin{abstract}
This paper presents an adaptive visualization for helping the needle insertion task for RF liver ablation under CT-fluoroscopy (CTfluoro) guidance. It shows slices of the 3D CT-volume that are resulting from the out-of-plane motion parameters along weighted isosurfaces in the convergence basin of the employed cost function. The visualization is coupled to the uncertainty in estimating the deformation of the liver and brings much more information than a single registered slice. We evaluated the proposed visualization with three experienced interventional radiologists and they pointed out that such visualization revealing the neighborhood with the belonging structures, like vessels and lesion spread, will help the needle guidance.
\end{abstract}

\section{Introduction}

Radiofrequency Ablation (RFA) is an interventional procedure applied to patients with unresectable primary liver tumors and metastases. The needle is percutaneously inserted into the lesion and can be performed eg. under CT-fluoro guidance. We would like to help this procedure by displaying high resolution contrasted data corresponding to the actual CT-fluoro slice in the intervention room when further needle insertion becomes difficult. In such cases we would like to enable an interface of triggering an automatic registration process and presenting e.g. the target lesion, neighborhood, vessel structures in the intervention room with the high quality of the contrasted pre-interventional CT-volume. However the deformation due to the breathing, the non-contrasted low resolution CT-fluoro makes it very hard to guarantee the validity of any deformable sliceto-volume registration. Attempts for a non-rigid 2D/3D registration employing the DEMONS algorithm [1] did not succeed even for very small deformations. Rigid slice-to-volume registration for similar applications has been reported in $[2,3]$. In [4] a visualization of a subvolume is proposed based on piecewise registration. In contrast to that approach, this paper aims to first obtain a solution to the global registration and then visualize a specific volume that encompasses its neighborhood. In this neighborhood, out-of-plane motion parameters samples on 
the isosurfaces of the minimized cost function are found using a line search strategy. The defined volume takes into account in which projection parameters the registration is most sensitive, and is obtained from the definition of slices defined by motion parameters on weighted isosurfaces. The visualization of a subvolume in the pre-interventional volume brings much more intuitive information to the interventional radiologist than one single slice. Therefore, the needle guidance toward the lesion is improved. In addition, this could result in less radiation dose for the patient and examiner.

\section{Materials and Methods}

Due to the noisy nature of the CT-fluoro slice, the needle presence and contrast differences, we are using a robust similarity measure to outliers and adaptive to discontinuities: the annealing M-estimator in conjunction with a GNC (graduated non-convexity) optimization [5] in order to compute the update parameters for the incremental pose $\mathbf{T}(\mathbf{x})$ in the volume that is composed with the current estimate $\widehat{\mathbf{T}}$ such that a cost function is minimized. The cost function $f_{\gamma}(\mathbf{x})$ is then defined as:

$$
f_{\gamma}(\mathbf{x})=\frac{\sum_{\mathcal{X}_{i}}\left(h_{\gamma}\left(d_{i}\right)\left|d_{i}\right|\right)}{\sum_{\mathcal{X}_{i}} h_{\gamma}\left(d_{i}\right)}
$$

with $h_{\gamma}\left(d_{i}\right)=1 /\left(1+d_{i}^{2} / \gamma\right)$ and $d_{i}=\mathcal{I}^{*}\left(\mathcal{X}_{i}\right)-\mathcal{I}\left(\widehat{\mathbf{T}} \mathbf{T}(\mathbf{x}) \mathcal{X}_{\mathbf{i}}\right)$. Here $\mathcal{I}^{*}\left(\mathcal{X}_{i}\right)$ is the intensity of the CT-fluoro slice at the pixel $\mathcal{X}_{i}$ and $\mathcal{I}\left(\widehat{\mathbf{T}} \mathbf{T}(\mathbf{x}) \mathcal{X}_{\mathbf{i}}\right)$ is the intensity in the CT-volume at the pixel $\mathcal{X}_{i}$ after being projected at the pose $\widehat{\mathbf{T}} \mathbf{T}(\mathbf{x})$. Since $h$ is continuous it is stable to changes of the difference conditions and $h_{\gamma}\left(d_{i}\right) / \sum_{\mathcal{X}_{i}} h_{\gamma}\left(d_{i}\right)$ is acting as a weight for $d_{i}$ penalizing outliers. The parameter $\gamma$ is set initially to a high value such that $h$ is strictly convex and is lowered according to a defined schedule.

For better spatial orientation, and to increase the confidence in the registration result, not only the minimizer of the registration function $f$ is visualized as a $2 \mathrm{D}$ view, but also other views "close" to the minimizer. The visualization of the $3 \mathrm{D}$ volume gives the intuition of slowly exploring the volume in the neighborhood (in real space) of the minimizer. For a given $\alpha \in \mathbb{R}^{+}$, an isosurface of the cost function $f$ defined in the equation (1) is a set $\mathcal{C}(\alpha) \subset \mathbb{R}^{6}$ given by $\mathcal{C}(\alpha)=\left\{\mathbf{x} \in \mathbb{R}^{6}: f_{\gamma}(\mathbf{x})=\alpha\right\}$. Now, let $\widetilde{\mathbf{x}} \in \mathbb{R}^{6}$ be the estimated minimizer. To find a certain number of parameters $\mathbf{x}_{i}$ around the minimizer, we use a line search in different directions for points on the isosurface $\mathcal{C}\left(f_{\gamma}(\widetilde{\mathbf{x}})+\varepsilon\right)$, where $\varepsilon$ defines the size of the neighborhood in parameter space. In section 3 we explain how we choose the directions of the line search and the $\varepsilon$ defining the size of the neighborhood. Each of these points correspond to a certain pose and therefore to a certain projection of the volume. We call these views cost-equivalent projections (CEPs). Once the minimizer of the function $f_{\gamma}(\mathbf{x})$ is identified and a number of samples on a given isosurface are determined, it is possible to define a trajectory interpolating these points. In order to visualize the volume smoothly, we first need to find an optimal trajectory from the minimizer through all of 
the isosurface sample points $\mathbf{x}_{i}$. Ideally, this trajectory follows the shortest path from the minimizer toward the isosurface, stays on the latter exactly, and aims for the minimizer again. As the isosurface itself is not analytically known, we use the sample points computed previously as waypoints, and rearrange them in a way that we visit each point once while following a trajectory of minimal length. We currently use the Euclidean norm as distance metric between parameters in $\mathbb{R}^{6}$. A smooth path is passing by the ordered points $\mathbf{x}_{i}$ defined above. We interpolate these points and the transformation matrices denote now the position and orientation of the virtual camera.

\section{$3 \quad$ Results}

The registration is performed with a coarse-to-fine strategy, starting at $64 \times 64$ and increasing to $128 \times 128$ and $256 \times 256$. Each time the estimated values are taken as initialization for the next resolution. The optimization procedure is started from the pose obtained by the DICOM Image Position Patient since the
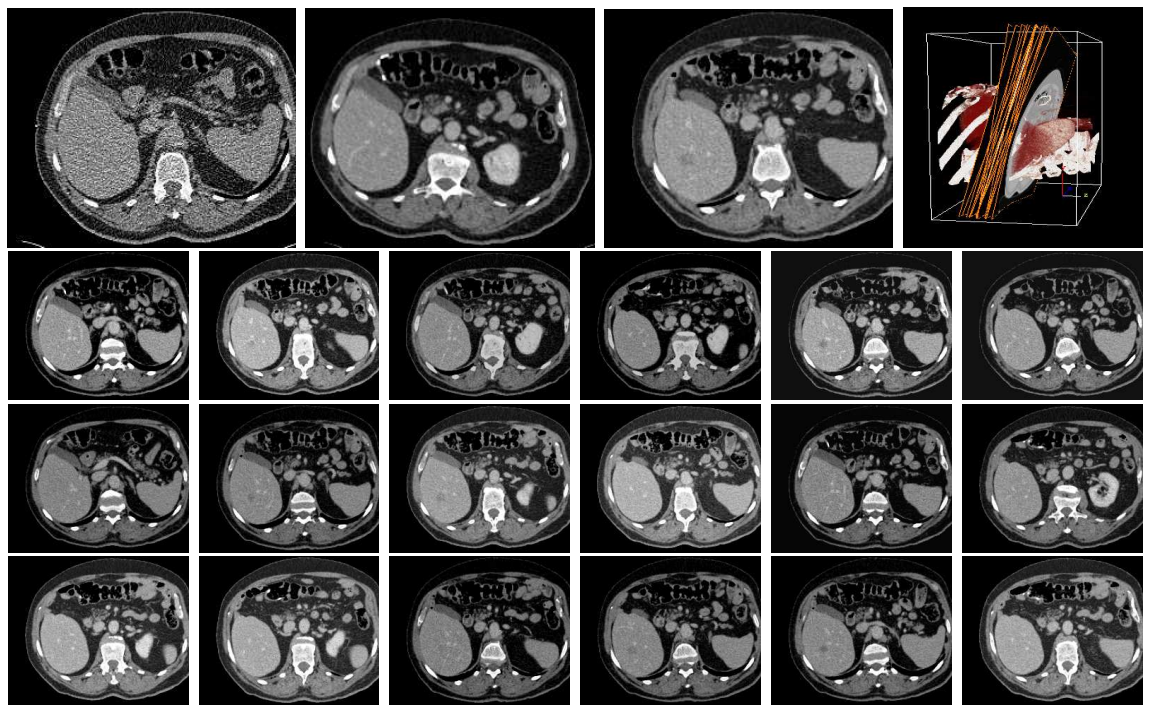

Fig. 1. First top row: the first image represents the CT-fluoro slice, the second one represents the initial pose in the volume and the third one represents the estimated minimum. Note that the lesion is not visible in the CT-fluoro slice. The forth image is showing a $3 \mathrm{D}$ view of the generated CEP's. The next rows are $2 \mathrm{D}$ views and represent CEPs at motions from out-of-plane parameters: 6 result from the 3 out-of-plane parameters in positive and negative direction, and 12 result from the combination of each of two parameters in positive and negative direction. The subvolume defined by the CEP's and the minimum slice is showed in a smooth animation. This example shows the possible convergence problems: the kidney is present in the initial pose and also in different CEP's whereas the liver shape does not significantly change 
patient is not moved from the table. The following experiments were run on a $\mathrm{PC}$ with $1.80 \mathrm{GHz}$ Intel Pentium processor and $1 \mathrm{~GB}$ of memory. The average time for the registration for the clinical images was $4 s$. The computation of the set of solutions on the isosurface took in average $15 \mathrm{~s}$. A solution set of 18 sampling points on the isosurface is used resulting from search directions obtained by combinations of out-of-plane parameters, i.e. translation along the $z$ axis, rotations about the $x$ and $y$ axis. Here, 6 CEPs result from the 3 outof-plane parameters in + and - direction, and 12 result from the combination of each of two parameters in + and - direction.

\subsection{Linking $\varepsilon$ with the Volume}

Based on results of the literature, e.g. [6], the deformation in the liver can reach up to $20 \mathrm{~mm}$ from the predicted rigid position, while the average across tissue yields about $6 \mathrm{~mm}$. Therefore, we would like the CEPs to represent an average movement between 6-7 mm. We start with an initial guess of $\varepsilon=0.25$ and adapt it until the average motion of all $3 \mathrm{D}$ points $\mathcal{X}_{i}$ considered in the volume between the minimum view and the views at the CEPs will be in this interval.

\subsection{Simulations Without Deformation}

To test the registration algorithm, we created a set of Ground-Truth (GT) slices simulating a CT-fluoro slice. We used from one patient a noisy reconstructed volume where we extracted at different poses including rotations and translations four slices. These slices were registered to the same pre-interventional volume but reconstructed without noise. An average RMS between the starting positions and the GT pose is about $35.75 \mathrm{~mm}$ for translation and $7.22^{\circ}$ for rotation. The results of the RMS calculations between registered noisy slices and the GT give an average RMS for translation of $0.65 \mathrm{~mm}$ and for rotation $0.03^{\circ}$.

\subsection{Simulations Including Deformation}

For deriving synthetic 2D slices that also include deformations, we are using two CT-volumes of one patient, the pre- and the post-interventional datasets. The pre-interventional CT-volume (the template volume) is elastically [7] registered to the post-interventional CT-volume (the reference volume). Since after the intervention, the patient is not any more able to achieve the full inspiration breath-hold as before the intervention, the registration of the pre-interventional toward the post-interventional scan is mimicking a possible expiration movement. The displacement field for the achieved deformation will map the voxels of the deformed volume into the pre-interventional scan. In this way by extracting axial slices of this deformed volume, adding noise, each of these slices represent hypothetical CT-fluoro slices. We are using six axial slices extracted every $1 \mathrm{~cm}$. Visual inspection on $3 \mathrm{D}$ views show that the deformation of the liver voxels is included by the estimated CEPs. 


\subsection{Experiments with real CT-fluoro slices}

Six routinely acquired datasets (Siemens Somatom Sensation 16) are used together with 3 CT-fluoro slices, which results in 18 examples. Figure 1 shows one example of the $2 \mathrm{D}$ views and a $3 \mathrm{D}$ view of all 18 estimated CEPs.

\subsection{Evaluation of Adaptive Visualization}

Evaluation of the CEP visualization versus the visualization of a single slice has been performed by three experienced interventional radiologists. In that setup we used though the results of the M-estimator, but the principles are the same. Two of the radiologists considered in all 18 times (100\%) the CEP visualization more valuable than one slice. One of them voted 11 times (61\%) for the CEP visualization. A single slice in the CT-volume will confirm or not the right position, but it misses further guidance and neighborhood assessment. On the other hand, this visualization ties up to the familiar view in the radiology.

\section{Conclusion}

We proposed an adaptive visualization that includes views of the CT-volume determined along flat directions of the out-of-plane motion parameters next to the minimizer where the optimization encounters uncertainty. The views are generated at poses that represent an average movement reported in the literature. In this way the deformation caused by the breathing should be included into the volume defined by the views. The result of the registration and the adaptive visualization were assessed by three experienced interventional radiologists on real CT-fluoro data. The evaluation outcome supports that such visualization in the intervention room enables the examiner to better orientate during the needle insertion.

\section{References}

1. Yaniv Z, Stenzel R, Cleary K, et al. A realistic simulation framework for assessing deformable slice-to-volume (CT-fluoroscopy/CT) registration. Proc SPIE. 2006;6141:116-23.

2. Birkfellner W, Figl M, Kettenbach J, et al. Rigid 2D/3D slice-to-volume registration and its application on fluoroscopic CT images. Med Phys. 2007;34(1):246-55.

3. Fei B, Duerk JL, Boll DT, et al. Slice-to-Volume Registration and its Potential Application to Interventional MRI-Guided Radio-Frequency Thermal Ablation of Prostate Cancer. IEEE Trans Med Imaging. 2003;22(4):515-25.

4. Micu R, Jakobs T, Zech C, et al. Evaluation of different subvolume visualizations in CT-Fluoroscopy guided RF Liver Ablation. Proc SPIE. 2007;6509:51-7.

5. Li SZ, Wang H, Soh WYC. Robust estimation of rotation angles from image sequences using the annealing M-Estimator. J Math Imaging Vis. 1998;8(2):181-192.

6. Clifford MA, Banovac F, Levy E, et al. Assessment of hepatic motion secondary to respiration for computer assisted interventions. Comp Aid Surg. 2002;7:291-9.

7. Modersitzki J. Numerical Methods for Image Registration. Oxford University Press; 2004. 